\title{
Automatic Airborne Laser Scanning Data Quality Control Procedure for Environmental Studies
}

\author{
Bartłomiej Kraszewski $\bowtie$, Żaneta Piasecka, Rafał Sadkowski, Krzysztof Stereńczak \\ Forest Research Institute, Department of Geomatics, Braci Leśnej 3, Sękocin Stary, 05-090 Raszyn, Poland, \\ phone: +48 22 7150325, fax: +48 22 7200397, e-mail: b.kraszewski@ibles.waw.pl
}

\begin{abstract}
Airborne laser scanning (ALS) technology delivers large amount of data collected from airborne level. These data are used for many different applications in forestry, civil engineering, environmental studies and others. To acquire the best possible results from the data, accuracy analysis is a necessary part of data processing chain. Therefore, considering the increasing interest worldwide in the use of laser scanning data, improving the quality control (QC) tools is a crucial pursuit.

This study underlines the possible error sources, summarises the existing QC knowledge for ALS data and proposes an optimised QC procedure. The procedure was implemented in selected applications and evaluated for three different environments, namely, forests, rural areas and croplands.

The proposed solution is almost fully automatic outside from the module that supports the operator in the classification examination. The workflow is scalable and can be expanded with new modules that enhance the functionality. The presented procedures can save up to 30 min of manual checks for every $1 \mathrm{~km}^{2}$ area.
\end{abstract}

\section{KeY WORDS}

ALS, automation, accuracy, density, quality, control

\section{INTRODUCTION}

Airborne laser scanning (ALS) is a rapid, highly accurate and efficient tool for capturing 3D topographic data over large areas. Initially, ALS was developed to provide accurate digital surface model (DSM) of the Earth's surface (Stereńczak et al. 2016). Very rapidly, it has become a tool used in forestry (Maltamo et al. 2014), geodesy (Haala and Kada 2010), geology (Wechsler et al. 2009), archaeology (Chase et al. 2011), environmental management (Vierling et al. 2008) and urban planning (Xiong et al. 2013). The ALS point cloud is an example of high-density vector spatial data. Each point in the point cloud is described by geometric and nongeometric information such as coordinates, intensity values, number of returns and others. Wu et al. (2011) mentioned that the first step in the ALS data quality control (QC) should be to validate the correctness of all the acquired information included in ALS data files.

The term 'quality' may have two contextual meanings in relation to ALS data as regards to 'quality assurance' (QA) and QC (Habib et al. 2009). QA is performed in advance of an acquisition and is concerned with management activities ensuring that a process, item or 
service achieves the quality the user requires (Renslow 2012). A key activity in the QA context is the calibration process that eliminates possible errors originating from the ALS system components. The most common errors of this kind are biases and random errors in the spatial and rotational offsets (Habib et al. 2010). During the calibration process, errors are calculated and considered in the final measurements in real projects.

The QC process is, in turn, conducted following a measurement mission or during the final data processing, with a view to verify the quality of the collected data. QC concerns an external (absolute) quality verification or an internal (relative) quality verification (Habib and Rens 2007). External data checking is based on comparison of ground control points with the ALS data. This analysis offers information regarding the integration of the collected data with the global coordinate system. The relative accuracy reports on data internal integrity are determined by reference to differences between single ALS flight strips.

The QA is dependent on provider or service precision during the calibration process. It is different from the QC focused on in this study that is correlated with the end-user needs and the capabilities of the data providers. ALS data need to be checked because assurances should be provided that the data has indeed been obtained with the required quality. More importantly, there is a need to ensure that future analyses will be trustworthy and carried out based on the best possible data.

The ALS data are mostly obtained in overlapping strips. Data overlapping allows the entire project area data to be connected into one geometrically consistent block. The quality of this connection is determined by relative accuracy by identifying the 3D shifts (on the X, $\mathrm{Y}$ and $\mathrm{Z}$ axes) between the strips. There are many possible error sources between two strips (Zhang et al. 2013) but a significant reason for an object to shift is the flight direction (Ahokas et al. 2004). Relative accuracy determination between ALS data strips is mainly carried out using an object that was mapped in overlapping areas of the strips. The most common methods for evaluation use roof ridge lines (Habib et al. 2008; Wężyk 2014) buildings (Ahokas et al. 2004) or roof faces (Filin 2003). In the case this type of feature is lacking, quality checks are performed using other planar surfaces such as roads (Maas 2002), parking lots or courts. When it comes to forest areas, canopy gaps or forest roads may be used as tie features. In many approaches, the segmentation procedure is used to extract the required surfaces to measure the shift between strips like the least-squares matching (LSM) (Gruen 1985), 2.5D grid comparison (Behan 2000), statistical computations (Latypov 2002) and profile view analysis (Bowen et al. 2002).

On the other hand, absolute accuracy assessments are performed to check how the ALS data were projected to a coordinate system. The accuracy is affected by the accuracy of scanning, positioning and navigation systems (Glennie 2007), the flight altitude of the sensor and platform, the characteristics of the scanned terrain, as well as the atmospheric conditions (Alharthy et al. 1999; Liu et al. 2008; Maas 2003). The horizontal and vertical accuracy of the ALS data are among the main factors used to determine the accuracy of the generated products such as DEMs (Liu 2011). The control methods of those errors are mainly based on the comparison of the features which can be located on ALS and measured in the field with Differential Global Positioning System (DGPS) or total station. Building roof planes or corners, and planar surfaces such as parking lots or courts were indicated by Maas (2003) as the most suitable for this purpose. Alharthy et al. (2004) proposed the use of drainage ditches for horizontal accuracy validations. Many authors mention that obtaining suitable objects for vertical accuracy evaluation is problematic (Bowen and Waltermire 2002; Alharthy et al. 2004).

One of the most important features that describe the details of the ALS data is the point density. In the United States Geological Survey (USGS) standard (Heidemann 2014), the point density is described as the Nominal Pulse Density (NPD) and is measured as the average number of pulses sent per specified areal unit, most often expressed as pts $/ \mathrm{m}^{2}$ (Wu et al. 2011; Rupnik et al. 2015). A point cloud with a higher density allows the creation of products with higher resolution (Lari et al. 2012). Methods for point density verification are mainly based on spherical neighbourhood analysis, eigenvalue analysis, Adaptive Cylinder Method (Lari and Habib 2012), box counting method (County 2003) and TIN-based (Triangulated Irregular Network) method (Rupnik et al. 2015). Methods using raster solutions should be considered where information about the point density in a specific area is needed. They are fast and give general information about the 
point density and area coverage. Solutions that analyse a point cloud in $3 \mathrm{D}$ space are excellent for determining the 3D distribution of points. We should also remember that within the project area, there might be areas without registered point clouds. These regions can be in a forest with a dense canopy, above water bodies, on roads or on objects made from non-reflecting or mirrored materials such as building roofs. An area over where no points are measured (Luethy and Ingensand 2004) is called a data void. These kinds of obstacles affect the quality of the DTM (Digital Terrain Model) and DSM (ALS data products) and should be separated, for example, by a method using image region growing proposed by $\mathrm{Wu}$ et al. (2011). We should also remember that the data void sizes and the point cloud density are important descriptors relating to data quality (Lari et al. 2012).

The point cloud from the ALS system contains points describing the surface of the Earth. To extract from these data the groups of points describing different classes of objects, a classification process should be performed. As a result of the classification, every point is assigned to one of a selected number of land cover classes. Most often, the classification process is done automatically and the errors are corrected manually. We can verify the classification accuracy in many ways, but the main problem is the availability of reference data. The referenced data could be the manually classified point cloud data (Kim and Sohn 2010), alternative classification methods (Wężyk 2014), orthophoto (Yan et al. 2012), aerial images (Antonarakis et al. 2008), 2D vector databases (Matikainen et al. 2009) or manually digitised vector layers (Tran et al. 2015). The most common metrics to describe classification accuracy are the overall accuracy (OA) and Kappa (Guo et al. 2015). These values are commonly used in image-based remote sensing classifications (Congalton 1991). There is a lack of sufficient automatic methods for verifying classification accuracy, leaving ALS data classification quality controllers many files to check. Manual control methods are time-consuming and as such, the point classification of the data is only checked visually to eliminate gross and systematic errors (Wężyk 2014). Therefore, classification accuracy is usually verified using randomly spread and representative samples (Yan et al. 2012).

The quality of ALS data is currently controlled in standard industrial processes. Various control processes are described in the QA reports (Groundpoint Technologies 2010; Dewberry 2014; URS Corporation 2012) created by the data providers or product quality standards (Polish Ministry of the Interior and Administration 2011) legislated by national institutions and they reflect the end-user needs. QA reports did not contain a uniform control procedure covering all QA aspects and were mainly based on manual workflows performed using commercial software. None of the proposed workflows dealt with the full data checking problem. Very few of the reports described the methods for performing semi-automatic or automatic checking process.

Therefore, in this study, we aimed to create a semiautomatic comprehensive ALS data control workflow that can be implemented by the end-users. The aims of the work detailed in this study are to review the existing methods for ALS data QC, to propose certain steps in the verification or quality checking procedure and to verify the results of the proposed QC procedure and discuss the possible benefits. A proposed procedure has been tested for selected land cover types.

\section{Materials AND Methods}

The developed ALS data quality checking workflow was tested in three regions with different land cover types. The first type presents an urban area located near a forest with small village constructions typical for Białowieża in Poland. The second was acquired for a forest area, and the last for an open space (a field). Every region had an area of 30 ha. The ALS data for each area were acquired in July 2015 using a Riegl LMS-Q680i system with a nominal point density of 6 points $/ \mathrm{m}^{2}$. A $25-\mathrm{cm}$ footprint size resulted from a flight altitude of $500 \mathrm{~m}$.

A schema of the proposed QC workflow is presented in Figure 1. The workflow assumes that checks on all levels are performed one after the other. If data do not pass any level, they are reported. Corrected data are checked again on all levels and accepted if the requirements are fulfilled. The first four levels are fully automatic, allowing for multiple repeat verification of large datasets without operator intervention. Only the last level (classification checking) is a semi-automatic and marks places where errors occurs. Indicated errors requiring manual visual verification. 


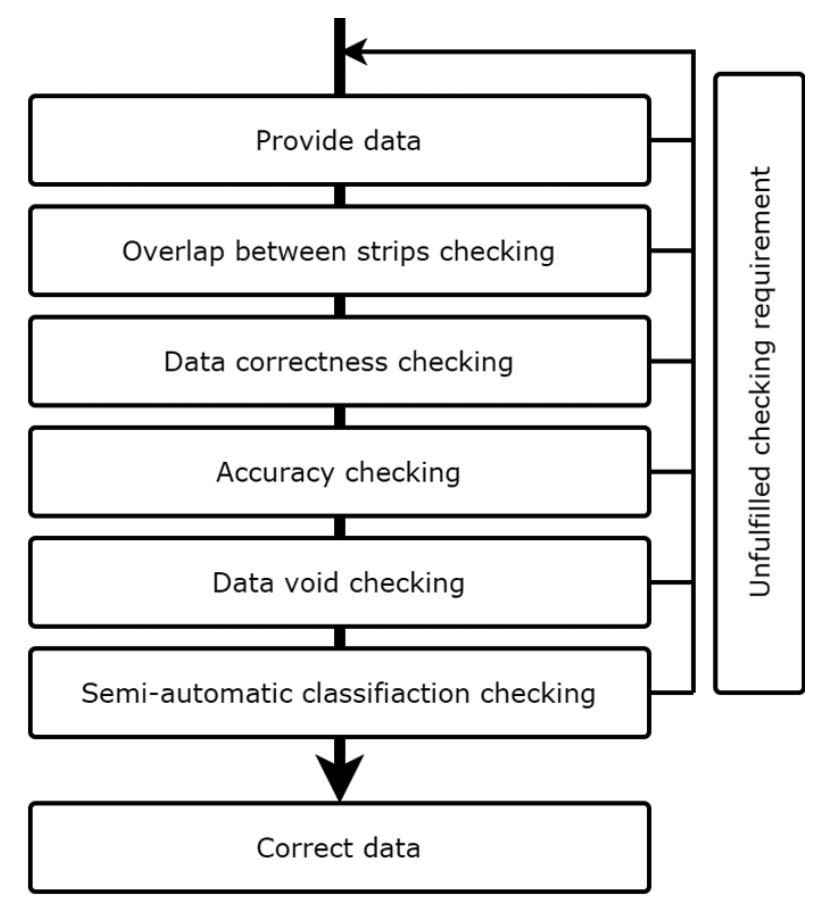

Figure 1. Workflow for the proposed ALS data quality control process

\section{Automatic checking of overlap between strips}

The points from the point cloud with the same ID are surrounded by a polygon using a concave hull, creating footprints of ALS strips. The generated footprints are used to check the overlap percentage between them. The overlap is calculated for all neighbouring strips.

\section{Automatic verification of data correctness}

The component checks if the file can be opened. In valid files, it verifies if each point has an RGB and intensity value or the assigned values do not exceed the expected range (e.g. 12 bits). The component checks also the density of the ALS point cloud in default $5 \times 5 \mathrm{~m}$ grid cells, but the size of the minimum unit can be adjusted to specific needs. In the first step, the first returned points from the point cloud are selected and a specified grid layer for the entire ALS area is created. Every pulse is counted and assigned to the grid cell. In the next step, the values per square metre are calculated and a raster of the density is generated.

The number of points in each cell from the density check is next used to calculate the coverage. The coverage informs about the percentage of the area that satisfies the defined point cloud density threshold.
In forested areas, it is very important to have a large proportion of points reflected from the tree crowns to better describe their structure. A low number of points describing tree crowns can be caused by strict filtration settings. The proposed method checks a parameter that describes the percentage of all points that should be measured between the first and last returns in tree stands of defined heights. In the proposed procedure, multi-return checking is performed in an area where the vegetation has a height greater than $25 \mathrm{~m}$. The number of returns between the first echo (FE) and the last echo (LE) should equate to $25 \%$ of the returns. In the proposed method, DSM is generated from a point cloud. The DSM serves to create regions from the pixels where the height value exceeds the $25-\mathrm{m}$ limit. Afterwards, the ALS points intersecting with the generated regions are selected and the number of points between the first and last returns is divided by the total number of points.

\section{Relative accuracy between strips}

The developed algorithm used two attributes of the point cloud: the class of the points and the ID of the strip. At the beginning, all ALS tiles are merged to single strips based on the IDs. Afterwards, from each strip, the points classified as buildings are extracted. The normals are then calculated for each point by analysing the eigenvectors and eigenvalues of a covariance matrix created from the nearest neighbours of the query point (Rusu 2010). Next the region-growing method is used to join together points representing a single surface based on the angle between the normal points. From among the grouped points, those occupying areas larger than $50 \mathrm{~m}^{2}$ are then selected. Analysis of the relative accuracy based on gabled roofs is done, and the clustered groups of points describing it have to be paired together. Groups describing roofs are joined if they intersect and their normal vectors are perpendicular, with a tolerance of $\pm 20^{\circ}$. Groups paired with each other (describing the gable roof) are used in the next step of the algorithm, where for every clustered point (representing single roof surface), a plane model is fitted using the Random Sample Consensus (RANSAC) algorithm (Fischler et al. 1981). The plane models in paired groups of the point are intersected and a linear model is calculated. The described algorithm for generating the linear model is used for every 
strip. Finally, the lines from each strip are compared to those near each other and then connected. The connected lines are divided into two sets running in the $\mathrm{N}-\mathrm{S}$ and $\mathrm{W}-\mathrm{E}$ directions, so that the translation between strips on the X- and Y-axes may be checked. The average distance for each line pairs is calculated before the RMSE (Root-mean-square error) in the X and $\mathrm{Y}$ directions for the data can be subsequently calculated. Ultimately, the operator receives the report as a shapefile containing lines with attributes describing the accuracy checks.

The previous method is dedicated for use in areas where there are buildings. For areas without welldefined human-related objects, planar surfaces can be used only for vertical accuracy assessment between overlapping strips. In this proposed method, first for each strip, the ground points (class 2 in LAS classification) are extracted. From the ground points, DTMs are generated for every strip and filtered with a median $(9 \times 9)$ filter. The filter window size can be modified. A filtered DTM is subtracted from the unfiltered DTM. Based on the subtracted raster, the mask containing 0-1 values is obtained (with 0 indicating non-flat areas and 1 indicating flat areas). Areas are defined as flat if the difference between both DTMs is lower than $0.05 \mathrm{~m}$. In the overlapping areas of the strip, raster with values of 1 (flat) are converted to a vector layer. In the meantime, a $2.5-\mathrm{m}$ grid net is generated and intersected with the resulting vector layer. Net tiles occupying less than $100 \%$ by flat areas are deleted. Among the generated 'flat' tiles, some (such as those representing road layers) are selected for further calculation. For the tiles belonging to each strip, the mean values of the pixels are collected. Finally, the difference between the strips' $\mathrm{Z}$ values is calculated.

\section{Absolute accuracy}

Checking the absolute accuracy can be performed in parallel with the relative accuracy by checking the position of building edges. However, the reference edges should be measured in the terrain using a total station. Next, the roof edge measurements are compared with the ridges assigned by the RANSAC algorithm. The workflow is the same as previously described for the relative accuracy. The edges need to be measured across and along the ALS flight lines in order to verify the accuracy in both directions.
When there are no buildings in the area of interest, a good solution for the vertical accuracy checking is the use of a DTM interpolated from the ALS data. This solution depends on the creation of cross-sectional profiles and includes four main steps. Firstly, control points on the ground are designed along different types of flat objects such as roads, fields, pavement and other similar objects. Ground control points are then measured by the total station or DGPS. In the next step of the process, a DTM is created from the ALS data. Finally, the height values from the DTM characterising the ground control points are extracted and compared with the height values of the field-based (referenced) measurements.

\section{Automatic checking for data voids}

The developed algorithm for checking data voids is very similar to the region-growing method presented by $\mathrm{Wu}$ et al. (2011). In contrast to $\mathrm{Wu}$ et al. (2011), our solution used the grid reclassification and GIS (Geographical Information System) tool for data void polygonisation.

First, the point cloud is transformed to a raster layer containing information on the number of points measured in each cell, such as the density information. The default density raster resolution is $1 \mathrm{~m}$, but can be changed by the user based on the point cloud density. Next, cells with values greater than 0 are changed to no data cells and the other cells are changed to data voids polygons. The number, size and distribution of the gaps can be a proxy for data quality and if needed, areas with large amounts of data gaps should be excluded from the analyses.

\section{Point cloud classification verifications}

The proposed semi-automated solution for ALS point cloud classification control is designed to identify potential error locations for later visual verification. The developed solution focused only on two classes of points: vegetation (classes 3-5) and buildings (class 6). First, in the algorithm, the height levels of the vegetation classes are checked (LAS classes $=3$ [low vegetation], 4 [medium vegetation], 5 [high vegetation]), and therefore, the point cloud is normalised using the DTM. Next, based on the normalised height and class of each point, an algorithm verifies how many points are classified correctly with respect to the height threshold value for each vegetation class. Finally, the proportion of 
points classified incorrectly among all points in a class is calculated.

Other possible errors are related to cases where vegetation is classified as a building, buildings are classified as vegetation, or (as the last common failure) vegetation is classified as part of a flat surface. In the proposed solution, the classes describing vegetation $(3,4,5)$ and buildings (6) are first selected from the point cloud. These selected points are then used to create two maximum height raster layers from points of the vegetation class and from the building points. The created rasters are then subtracted from each other. No data cells in the difference raster means that buildings and vegetation do not coincide with each other. If the cell value is greater than 0 , then vegetation has been above the buildings. It is a situation usually noticed in forests when the points describing the trees are classified as buildings. On the other hand, if a value is below 0 , then the vegetation has been placed under a building; it can be true in some circumstances (walls of buildings classified as vegetation). In the final step, the proposed algorithm requires the operator to decide if the error is relevant or not. The operator should check all marked locations manually.

\section{Results}

The proposed ALS data control workflow was implemented within a specially created package of tools called LasControl. After each step in the process, a report with the results of the data control was generated. Values exceeding the limits defined by the user were marked red as potentially incorrect as shown in Figure 2. In some cases, the algorithm generated raster maps presenting the point cloud density for each analysed sheet to support the operator in checking the classification.
We tested all steps of the verification tool on three regions (forest, rural and open sites), seeking to determine which of them was appropriate in relation to land cover types.

For each test site, the point cloud density was checked. The most homogenous results were acquired for the crop field. This was expected because there is no other object on the ground apart from small vegetation. This land-use type had the highest density of $11.2 \mathrm{pts} / \mathrm{m}^{2}$. The lowest point cloud density $\left(9.8 \mathrm{pts} / \mathrm{m}^{2}\right)$ was noticed for the rural region, where a lot of different man-made objects as well as a variety of vegetation were noticed. In the forest, the point cloud density was $11 \mathrm{pts} / \mathrm{m}^{2}$.

The forest area and crop sites were covered by a point cloud with density greater than $6 \mathrm{pts} / \mathrm{m}^{2}$ in more than $95 \%$ of the area $(96.4 \%$ and $95.3 \%$, respectively). Only the rural site had lower coverage (91.4\%) resulting from the presence of water bodies on the site. The water reservoir absorbed the laser beam signal and the points were not collected from its surface. The location of the water reservoir is clearly visible in white on the point cloud density map presented in Figure 3.

For all the checked files, the RGB and intensity attributes of the point cloud were correctly written. The point cloud returns between the first and last in all sites accounted for over $50 \%$ of all returns.

The test site had regions without measurement points. The largest summary area $\left(11,649 \mathrm{~m}^{2}\right)$ without points was located in the rural area as a result of water bodies located in the site. The largest single no-data area in this site was $8,570 \mathrm{~m}^{2}$. A small area with nodata occupied $1,219 \mathrm{~m}^{2}$ in the crop site. In the rural site, a single group of no-data values occurred, which appeared at the place where the flight strips were joined. On all sites, the no-data regions ranged between 0.5 and $3.0 \mathrm{~m}^{2}$.

\begin{tabular}{|c|c|l|c|c|c|c|c|c|}
\hline & Is Correct? & \multicolumn{1}{|c|}{ Name File } & $\begin{array}{c}\text { Density } \\
{\left[\mathrm{p} / \mathrm{m}^{2}\right]}\end{array}$ & $\begin{array}{c}\text { Coverage } \\
{[\%]}\end{array}$ & $\begin{array}{c}\text { Classification } \\
{[\%]}\end{array}$ & Intenisty & RGB & $\begin{array}{c}\text { Return } \\
{[\%]}\end{array}$ \\
\hline 1 & 1 & FOREST_N-34-120-D-c-3-2-2-4 & 11.0 & 96.4 & 99.9 & $\mathrm{~T}$ & $\mathrm{~T}$ & 52.8 \\
\hline 2 & 0 & FOREST_N-34-120-D-c-4-4-3-4 & 10.4 & 94.7 & 99.8 & $\mathrm{~T}$ & $\mathrm{~T}$ & 55.3 \\
\hline 3 & 0 & CITY_N-34-120-D-c-4-1-2-4 & 9.8 & 91.4 & 98.0 & $\mathrm{~T}$ & $\mathrm{~T}$ & 58.6 \\
\hline 4 & 1 & CITY_N-34-120-D-c-3-1-1-3 & 10.3 & 97.0 & 99.4 & $\mathrm{~T}$ & $\mathrm{~T}$ & 51.5 \\
\hline 5 & 1 & GROUND_N-34-120-A-c-4-4-3-1 & 11.2 & 95.3 & 99.6 & $\mathrm{~T}$ & $\mathrm{~T}$ & 75.0 \\
\hline
\end{tabular}

Figure 2. An example of a report generated for the tested data 


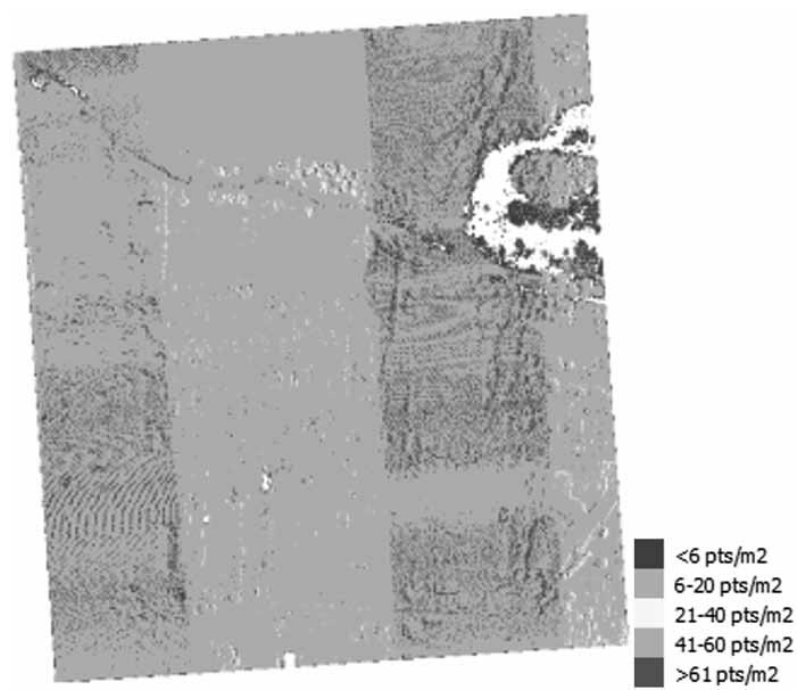

Figure 3. Density map for the rural area $(5 \mathrm{~m}$ raster resolution)

The horizontal and vertical relative accuracy checking between strips could be performed only on the rural site due to the presence of buildings. The accuracy between the strips in this area was $4 \mathrm{~cm}$ horizontally and $15 \mathrm{~cm}$ vertically. For the forest and crop site, only the vertical accuracy could be checked because on this location, there were no objects that could be used as a reference. The vertical accuracy for these sites reached $1 \mathrm{~cm}$.

Using the semi-automatic method for classification checking, only two sites were found with errors in the point classifications. For the forest sites, some points describing trees were classified as buildings, and in the rural area, some points were classified as trees when, in fact, they were measured on buildings. In the second case, the points were incorrectly classified almost always on building walls.

\section{Discussion AND CONCLUSION}

Tests performed on the example sheets of a point cloud showed that all types of terrain may be subjected to the checks involving strip coverage, correctness and data voids. Automatic accuracy checks can be applied to all data acquired in different land cover, although only the relative and absolute accuracy of the $\mathrm{Z}$ coordinates can be checked on flat areas using the raster solution. To horizontal accuracy evaluation in a point cloud should be visible ridges of buildings that are relatively measured on two strips or in the terrain. The best solution is to measure building's ridges arranged perpendicularly to each other. Places suitable for this may occur in either urban or rural areas. The final control checks are dedicated to just two types of locations in which two implemented cases can arise, such as in an area where the points reflected from buildings are classified as vegetation and in forest areas where the points acquired between trees can be marked as in the building class.

To acquire good quality of analyses, the ALS data need to be correctly collected and processed. Correctness can be determined following a control evaluation. The QC and checking process should be a standard procedure connected with provider post-processing to confirm the quality of the prepared data. The end-user should also be aware of the data quality. The best solution is an easy control procedure allowing for data checking using automated and semi-automated methods. Automation minimises the time needed for visual and manual interpretation by an operator. As a consequence, the time needed for data checking and the elimination of post-processing errors that cause erroneous results is reduced. The correct workflow of the consecutive steps allows the data control to focus on moving from the general to the detailed parameters of a point cloud. A standard control protocol makes the data acquisition a more stable process and allows for faster data use.

In our solution, we first performed a procedure that was suggested by Wu et al. (2011) to verify the correctness of the ALS files. The methods we used were inspired by the methods described in the literature and included a point density check in a regular raster grid (County 2003) and absolute accuracy checking using roof ridges (Ahokas et al. 2004; Habib et al. 2008; Wężyk 2014). Our solution can save up to $30 \mathrm{~min}$ of manual checking for each $1 \mathrm{~km}^{2}$ area. More time can be gained in the case where automatic checking procedures not requiring operator intervention beyond the definition of control parameters can be applied. Checking the point cloud classifications only requires the involvement of operators in the visual interpretation of the sets or some percentage of the data. Automation of the data verification is very difficult because it is hard 
to implement a solution that captures all of the errors. The developed solution only highlights locations where certain obvious errors can occur, thereby supporting the person supervising the process. Automatic tools are recommended for projects covering terrain areas of more than $10 \mathrm{~km}^{2}$, where up to a few days of verification work can be saved.

A very important module in data control is the determination of void data areas. The occurrence of these areas influences the quality of the DTM interpolation. In cases with forested areas, the point cloud density is very low and the accuracy of the DTM interpolation is lower than in rural or urban locals. For forest sites with no measurements, we can conclude when the data was acquired. Data void areas may also be used to find the places where existing land cover is less laser beam reflective, such as in water reservoirs.

We presented procedures used to check multiple datasets from real projects. The workflow allows to detect and eliminate all of the defined errors. This is done in close cooperation with a data provider having full access to the description of the procedures. The control rules and methods ensure transparent cooperation, and data improvements, in our case, were easy and without unnecessary disputes or inconsistencies. The presented solution is fully automatic outside the module that may be supported by the operator for checking classifications. The solution workflow is scalable and can be expanded to include new modules that enrich the functionality.

Considering the increased interest in the use of laser scanning data, procedures and tools for QC are needed worldwide. Such standard procedures and tools presented here can be used in many different projects where the data are acquired for multiple purposes. The tools allow the user to select the modules needed for the purposes and to define the checking parameters.

\section{ACKNOWLeDGements}

This work was financially supported by the Project LIFE + ForBioSensing PL Comprehensive monitoring of stand dynamics in Białowieża Forest supported with remote sensing techniques and was co-funded by Life Plus (contract number LIFE13 ENV/PL/000048) and The National Fund for Environmental Protection and Water Management in Poland (contract number 485/2014/WN10/OP-NM-LF/D).

\section{References}

Ahokas, E., Kaartinen, H., Hyyppä, J. 2004. A quality assessment of repeated airborne laser scanner observations. International Archives of Photogrammetry, Remote Sensing and Spatial Information Sciences, 35, 237-242.

Alharthy, A., Bethel, J., Mikhail, E. M. 2004. Analysis and accuracy assessment of airborne laserscanning system. International Archives of Photogrammetry, Remote Sensing and Spatial Information Sciences, 35, 144-149.

Antonarakis, A.S., Richards, K.S., Brasington, J. 2008. Object-based land cover classification using airborne LiDAR. Remote Sensing of Environment, 112 (6), 2988-2998. DOI: 10.1016/j.rse.2008.02.004

Baltsavias, E.P. 1999. Airborne laser scanning: basic relations and formulas. ISPRS Journal of photogrammetry and remote sensing, 54 (2), 199-214. DOI: 10.1016/S0924-2716(99)00015-5

Behan, A. 2000. On the matching accuracy of rasterised scanning laser altimeter data. International Archives of Photogrammetry and Remote Sensing, 33 (B2), 75-80.

Bowen, Z.H., Waltermire, R.G. 2002. Evaluation of light detection and ranging (LIDAR) for measuring river corridor topography. JAWRA Journal of the American Water Resources Association, 38 (1), 33-41.

Chase, A.F. et al. 2011. Airborne LiDAR, archaeology, and the ancient Maya landscape at Caracol, Belize. Journal of Archaeological Science, 38 (2), 387-398. DOI: $10.1016 /$ j.jas.2010.09.018

Congalton, R.G. 1991. A review of assessing the accuracy of classifications of remotely sensed data. Remote Sensing of Environment, 37 (1), 35-46. DOI: 10.1016/0034-4257(91)90048-b

Congalton, R.G., Green, K. 2008. Assessing the accuracy of remotely sensed data: principles and practices. CRC press.

County, K. 2003. LiDAR digital ground model point density, KGIS Center, Seattle, WA. Available at http://www5.kingcounty.gov/sdc/raster/elevation/ 
LiDAR_Digital_Ground Model_Point Density. html/

Dewberry, 2014. LiDAR Quality Assurance for Oahu LiDAR Produced for National Oceanic and Atmospheric Administration. Available at https://coast. noaa.gov/htdata/lidar1_z/geoid12a/data/3655/supplemental/hi2013_noaa_oahu_lidarreport_m3655. pdf

Filin, S. 2003. Analysis and implementation of a laser strip adjustment model. International Archives of Photogrammetry and Remote Sensing, 65-70.

Fischler, M.A., Bolles R.C. 1981. Random sample consensus: a paradigm for model fitting with application to image analysis and automated cartography. Communications of the ACM, 24 (6), 381-395.

Groundpoint Technologies. 2010. Accuracy assessment and quality control report for the Milwaukee, WI Project Area. Available at http://www.county.milwaukee.gov/ImageLibrary/User/bshaw/LiDARQAQC_MilwaukeeWI_final_01.pdf

Glennie, C. 2007. Rigorous 3D error analysis of kinematic scanning LIDAR systems. Journal of Applied Geodesy, 1 (3), 147-157.

Gruen, A. 1985. Adaptive least squares correlation: a powerful image matching technique. South African Journal of Photogrammetry, Remote Sensing and Cartography, 14 (3), 175-187.

Guo, B., Huang, X., Zhang, F., Sohn, G., 2015. Classification of airborne laser scanning data using JointBoost. ISPRS Journal of Photogrammetry and Remote Sensing, 100, 71-83. DOI: 10.1016/j.isprsjprs.2014.03.004

Haala, N., Kada, M. 2010. An update on automatic 3D building reconstruction. ISPRS Journal of Photogrammetry and Remote Sensing, 65 (6), 570-580. DOI: 10.1016/j.isprsjprs.2010.09.006

Habib, A., Bang, K.I., Kersting, A.P., Chow, J. 2010. Alternative methodologies for LiDAR system calibration. Remote Sensing, 2 (3), 874-907. DOI: 10.3390/ rs2030874

Habib, A., Bang, K.I., Kersting, A.P., Lee, D.C. 2009. Error budget of LiDAR systems and quality control of the derived data. Photogrammetric Engineering and Remote Sensing, 75 (9), 1093-1108. DOI: 10.14358/PERS.75.9.1093

Habib, A.F., Kersting, A.P., Ruifang, Z., AlDurgham, M., Kim, C., Lee, D.C. 2008. LiDAR strip adjustment using conjugate linear features in overlapping strips. International Archives of the Photogrammetry, Remote Sensing and Spatial Information Sciences, 37, 385-390.

Habib, A.F., Rens, J.V. 2007. Quality assurance and quality control of Lidar systems and derived data. In: Advanced Lidar Workshop, University of Northern Iowa.

Heidemann, H.K. 2014. Lidar base specification version 1.0: US Geological survey techniques and methods. Book, 11, 63.

Kim, H.B., Sohn, G. 2010. 3D classification of powerline scene from airborne laser scanning data using random forests. International Archives of Photogrammetry and Remote Sensing, 38, 126-132.

Lari, Z., Habib, A. 2012. Alternative methodologies for the estimation of local point density index: Moving towards adaptive LiDAR data processing. International Archives of Photogrammetry, Remote Sensing and Spatial Information Sciences, 39, 127-132.

Latypov, D. 2002. Estimating relative lidar accuracy information from overlapping flight lines. ISPRS Journal of Photogrammetry and Remote Sensing, 56 (4), 236-245. DOI: 10.1016/S09242716(02)00047-3

Liu, X. 2011. Accuracy assessment of LiDAR elevation data using survey marks. Survey Review, 43 (319), 80-93.

Liu, Z.S., Liu, B.Y., Wu, S.H., Li, Z.G., Wang, Z.J. 2008. High spatial and temporal resolution mobile incoherent Doppler lidar for sea surface wind measurements. Optics letters, 33 (13), 1485-1487.

Luethy, J., Ingensand, H. 2004. How to evaluate the quality of airborne laser scanning data. Proceedings of NATSCAN-Conference on Laser-Scanners for Forest- and Landscape Assessment, Freiburg, Germany, October 3-6, 2004. International Archives of Photogrammetry and Remote Sensing, 36 (8/W2), 313-317.

Maas, H.G. 2003. Planimetric and height accuracy of airborne laserscanner data: User requirements and system performance. Proceedings of Photogrammetric Week, Vol. 49, 117-125.

Maas, H.G. 2002. Methods for measuring height and planimetry discrepancies in airborne laserscanner data. Photogrammetric Engineering and Remote Sensing, 68 (9), 933-940. 
Maltamo, M., Næsset, E., Vauhkonen, J. 2014. Forestry applications of airborne laser scanning. Concepts and case studies. Managing for Ecosystems, 27, 2014.

Matikainen, L., Hyyppä, J., Kaartinen, H. 2009. Comparison between first pulse and last pulse laser scanner data in the automatic detection of buildings. Photogrammetric Engineering and Remote Sensing, 75 (2), 133-146.

Polish Ministry of the Interior and Administration. 2011. Ordinance of the Minister of Interior and Administration of 3 November 2011 on aerial and satellite imagery databases and orthophotomaps and numerical terrain models.

Renslow, M.S. 2012. Manual of Airborne Topographic LiDAR. American Society for Photogrammetry Remote Sensing, Bethesda, Maryland.

Rupnik, B., Mongus, D., Žalik, B. 2015. Point Density Evaluation of Airborne LiDAR Datasets. Journal of Universal Computer Science, 21(4), 587-603. DOI: 10.3217/jucs-021-04-0587

Rusu, R.B. 2010. Semantic 3d object maps for everyday manipulation in human living environments. $K I$ KünstlicheIntelligenz, 24(4), 345-348.

Stereńczak, K., Ciesielski, M., Bałazy, R., ZawiłaNiedźwiecki, T. 2016. Comparison of various algorithms for DTM interpolation from LIDAR data in dense mountain forests. European Journal of Remote Sensing, 49, 599-621.

Tran, G., Nguyen, D., Milenkovic, M., Pfeifer, N. 2015. Potential of Full Waveform Airborne Laser Scanning Data for Urban Area Classification-Transfer of Classification Approaches Between Missions. The International Archives of Photogrammetry, Remote Sensing and Spatial Information Sciences, 40 (7), 1317-1323.

Vierling, K.T., Vierling, L.A., Gould, W.A., Martinuzzi, S., Clawges, R.M. 2008. Lidar: shedding new light on habitat characterization and modeling. Frontiers in Ecology and the Environment, 6(2), 90-98. DOI: 10.1890/070001

URS Corporation. 2012. Independent LiDAR Quality Control Report - Calvert Country Area of Interest. Available at http://webmaps.esrgc.org/LiDAR/portal/client/download/Metadata/Calvert/Final $\% 20$ Calvert\%20LiDAR\%20QA\%20Report.pdf

Wechsler, N., Rockwell, T.K., Ben-Zion, Y. 2009. Application of high resolution DEM data to detect rock damage from geomorphic signals along the central San Jacinto Fault. Geomorphology, 113 (1), 82-96.

Wężyk, P. 2014. Podręcznik dla uczestników szkoleń z wykorzystaniem produktów LiDAR. Głowny Urząd Geodezji i Kartografii, Warszawa.

Wu, J., Yao, W., Chi, W., Zhao, X. 2011. Comprehensive quality evaluation of airborne lidar data. International Symposium on Lidar and Radar Mapping Technologies, 828604-828604. International Society for Optics and Photonics.

Xiong, X., Adan, A., Akinci, B., Huber, D. 2013. Automatic creation of semantically rich $3 \mathrm{D}$ building models from laser scanner data. Automation in Construction, 31, 325-337. DOI: 10.1016/j.autcon.2012.10.006

Yan, W.Y., Shaker, A., Habib, A., Kersting, A.P. 2012. Improving classification accuracy of airborne LiDAR intensity data by geometric calibration and radiometric correction. ISPRS Journal of Photogrammetry and Remote Sensing, 67, 35-44. DOI: http://dx.doi.org/10.1016/j.isprsjprs.2011.10.005

Zhang, Y.J., Xiong, X.D., Hu, X.Y. 2013. Rigorous LiDAR Strip Adjustment with Triangulated Aerial Imagery. ISPRS Annals of Photogrammetry, Remote Sensing and Spatial Information Sciences, II-5/W2, 361-366. DOI: 10.5194/isprsannals-II5-W2-361-2013 